\title{
Telmisartan ameliorates insulin sensitivity by activating the AMPK/SIRT1 pathway in skeletal muscle of obese $d b / d b$ mice
}

\author{
Asuka Shiota ${ }^{1,2}$, Michio Shimabukuro ${ }^{1,2^{*}}$, Daiju Fukuda ${ }^{1,2}$, Takeshi Soeki $^{2}$, Hiromi Sato ${ }^{3}$, Etsuko Uematsu², \\ Yoichiro Hirata ${ }^{2}$, Hirotsugu Kurobe ${ }^{4}$, Norikazu Maeda ${ }^{5}$, Hiroshi Sakaue ${ }^{3}$, Hiroaki Masuzaki ${ }^{6}$, lichiro Shimomura ${ }^{5}$ \\ and Masataka Sata ${ }^{2}$
}

\begin{abstract}
Background: Telmisartan is a well-established angiotensin II type 1 receptor blocker that improves insulin sensitivity in animal models of obesity and insulin resistance, as well as in humans. Telmisartan has been reported to function as a partial agonist of the peroxisome proliferator-activated receptor (PPAR) $\gamma$, which is also targeted by the nicotinamide adenine dinucleotide (NAD)-dependent deacetylase (SIRT1). Here, we investigated the pathways through which telmisartan acts on skeletal muscle, in vitro as well as in vivo.

Methods: Nine-week-old male $d b / d b$ mice were fed a $60 \%$ high-fat diet, with orally administrated either vehicle (carboxymethyl-cellulose, CMC), $5 \mathrm{mg} / \mathrm{kg}$ telmisartan, or $5 \mathrm{mg} / \mathrm{kg}$ telmisartan and $1 \mathrm{mg} / \mathrm{kg}$ GW9662, a selective irreversible antagonist of PPARY, for 5 weeks. Effects of telmisartan on Sirt1 mRNA, AMPK phosphorylation, and NAD $+/ \mathrm{NADH}$ ratio were determined in $\mathrm{C} 2 \mathrm{C} 12$ cultured myocytes.

Results and discussion: Telmisartan treatment improved insulin sensitivity in obese $d b / d b$ mice fed a high-fat diet and led to reduction in the size of hypertrophic pancreatic islets in these mice. Moreover, in vitro treatment with telmisartan led to increased expression of Sirt1 mRNA in C2C12 skeletal muscle cells; the increase in Sirt1 mRNA in telmisartan-treated C2C12 myoblasts occurred concomitantly with an increase in AMPK phosphorylation, an increase in NAD+/NADH ratio, and increases in the mRNA levels of PGC1a, FATP1, ACO, and GLUT4.
\end{abstract}

Conclusions: Our results indicate that telmisartan acts through a PPARY-independent pathway, but at least partially exerts its effects by acting directly on skeletal muscle AMPK/SIRT1 pathways.

Keywords: Adiponectin, AMP-activated protein kinase, Obesity, Peroxisome proliferator-activated receptor- $\gamma$, SIRT1

\section{Introduction}

The nicotinamide adenine dinucleotide (NAD)-dependent deacetylase (SIRT1) has been reported to be involved in protection against metabolic disorders as well as in enhancing life span [1]. Evidence suggests that SIRT1 also plays a fundamental role in the metabolism and differentiation of skeletal muscle cells [2], and transgenic or knockout Sirt1 mouse models have implicated the protein in protection of skeletal muscle against oxidative stress $[3,4]$. It has

\footnotetext{
* Correspondence: mshimabukuro-ur@umin.ac.jp

'Department of Cardio-Diabetes Medicine, University of Tokushima Graduate School of Health Biosciences, 3-18-15 Kuramoto, Tokushima 770-8503, Japan ${ }^{2}$ Department of Cardiovascular Medicine, University of Tokushima Graduate School of Health Biosciences, Tokushima, Japan

Full list of author information is available at the end of the article
}

been speculated that SIRT1 plays an important role in the regulation of transcriptional networks in various critical metabolic processes [1]. The SIRT1 signal seems to be mediated by an increase in fatty acid oxidation by activation of AMP-activated protein kinase (AMPK) in skeletal muscle [5]. The AMPK activators metformin [6] and A-769662 [7], as well as resveratrol [8], a polyphenolic SIRT1 activator, ameliorate insulin resistance in animals given a high-fat diet.

Telmisartan is a well-established angiotensin II type 1 receptor (AT1) blocker that improves insulin sensitivity in rodents that have received high-fat-containing diets [9-12], as well as in diabetic [12] and nondiabetic patients [13]. Telmisartan has been reported to function 
as a partial agonist of the peroxisome proliferatoractivated receptor (PPAR) $\gamma$, a member of the ligandactivated nuclear receptor superfamily that is expressed at high levels in adipose tissue [14]. PPARy regulates genes that modulate lipid utilization and storage, lipoprotein metabolism, adipocyte differentiation, and insulin action [14]. Thus, it is possible that the antidiabetic effects of telmisartan depend largely on PPAR $\gamma$ dependent mechanisms [15]. PPAR $\gamma$ and the PPAR $\gamma$ coactivator (PGC)-1 $\alpha$, which play key roles in various metabolic disorders, are also known to be targeted by SIRT1 [16,17], but the effects of telmisartan on SIRT1and PPAR $\gamma$-signaling remain unclear.

In the present study, we investigated whether telmisartan acts on skeletal muscle through an AMPK/SIRT1 pathway in skeletal muscle.

\section{Materials and methods}

\section{Animals and experimental protocol}

Study protocols were approved by the Committee on Animal Research, the University of Tokushima, and have been done on the basis of ethical principles and guidelines for experiments on animals (http://freedownload.is/ pdf/ethical-principles-and-guidelines-for-experimentson-animals-22328952.html). Nine-week-old male $d b / d b$ mice (Charles River Laboratories Japan Inc. Tokyo, Japan) were fed a high-fat diet (HFD-60, Oriental Yeast Co., Ltd., Tokyo) ad libitum for 5 weeks. HFD-60 contains energy content $62 \%$ fat (lard 33, milk-casein 26, cornstarch 16 g/100 g•diet; fatty acid 16:0 24.4, C18:0 13.8, C:18:1 41.8, 18:2(n-6) $12.0 \mathrm{~g} / 100 \mathrm{~g}$ of total fatty acids), $18 \%$ protein and $20 \%$ carbohydrate, compatible with the AIN-93 G recommendations [18]. $d b / d b$ mice were separated into 3 groups and were orally administrated either vehicle (carboxymethyl-cellulose, CMC), $5 \mathrm{mg} / \mathrm{kg}$ telmisartan, or $5 \mathrm{mg} / \mathrm{kg}$ telmisartan and $1 \mathrm{mg} / \mathrm{kg}$ GW9662 (Sigma, St. Louis, MO), a selective irreversible antagonist of PPAR $\gamma$, once a day, for 5 weeks. Age-matched male wild type $+/+$ mice (Charles River Laboratories Japan) fed a normal diet, containing energy content $10 \%$ fat, $14 \%$ protein and 76\% carbohydrate (AIN-93 M, Oriental Yeast Co. Ltd.) were used as a control group.

We defined the dose usage of telmisartan and GW9662 as follows. (1) telmisartan ( $5 \mathrm{mg} / \mathrm{kg} /$ day). For hypertensive patients, telmisartan is orally administered at the dose of $20-160 \mathrm{mg}$ per day, which is equivalent to $\approx 0.3-3 \mathrm{mg} / \mathrm{kg} /$ day. A pharmacokinetic study showed that steady state Cmax of telmisartan was $28.3 \mathrm{ng} / \mathrm{ml}$ at $20 \mathrm{mg}$ and $592 \mathrm{ng} / \mathrm{ml}$ at $120 \mathrm{mg}(\approx 0.06-1.15 \mu \mathrm{mol} / \mathrm{L})$ in normotensive elderly subjects [19]. We had preliminary confirmed telmisartan show a PPAR- $\gamma$ agonistic effect at doses of 0.5-10 $\mu \mathrm{M}$ in HEK293 cells (data not shown). Combined, we selected the dose of $5 \mathrm{mg} / \mathrm{kg} /$ day to obtain a PPAR $-\gamma$ agonistic effect at a clinical dose. (2)
GW9662 (1 mg/kg/day). We determined the dose of GW9662 following Goyal et al [20]. They showed that the protective effects of telmisartan $(10 \mathrm{mg} / \mathrm{kg} /$ day $)$ on myocardial infarction model in experimental diabetes could be modulated by down-regulated PPAR- $\gamma$ expression by $1 \mathrm{mg} / \mathrm{kg} /$ day of GW9662 [20].

Body weight, food intake, systolic blood pressure and blood glucose were measured weekly. Blood pressure was measured using a tail-cuff system (Softron, Co., Tokyo) and glucose was determined by a glucose oxidase test. Mice were housed in a light- and temperaturecontrolled room, with a 12-hour light/dark cycle.

At the end of the experimental period, an insulin tolerance test (ITT) was performed. Mice were given an intraperitoneal injection of $0.75 \mathrm{U} / \mathrm{kg}$ insulin, after which blood glucose concentrations were measured at 0,15 , 30, 60, and $120 \mathrm{~min}$. Moreover, at the end of the experimental period, serum, and subcutaneous and visceral fat, were collected and stored at $-80^{\circ} \mathrm{C}$ until assayed. Insulin was determined by a mouse insulin ELISA kit (AKRIN011 T, Shibayagi, Gunma, Japan).

Distribution of adipocyte size was determined as previously described [21]. Briefly, adipocytes isolated from visceral and subcutaneous adipose tissue were fixed with $2 \%$ osmium tetroxide and passed through a $250-\mu \mathrm{m}$ nylon filter to remove the fibrous elements, after which the cells were washed extensively with isotonic saline. 10000 cells were analyzed using the Coulter Multisizer III (Beckman Coulter, High Wycombe, England). Paraffin-embedded serial sections of pancreata $(5-\mu \mathrm{m}$ thickness) were stained for Hematoxylin and Eosin (HE) and indirect insulin immunostaining [22].

\section{Cell culture}

C2C12 myoblasts were cultured in Dulbecco's modified Eagle's medium (DMEM; Sigma) with $4.5 \mathrm{~g} / \mathrm{L}$ glucose supplemented with $10 \%$ fetal bovine serum (FBS; Sigma), 100 $\mathrm{U} / \mathrm{mL}$ penicillin, and $100 \mu \mathrm{g} / \mathrm{mL}$ streptomycin as previously described [23]. One day after $\mathrm{C} 2 \mathrm{C} 12$ myoblasts reached confluence (day 0), cells were induced to differentiate into myocytes by replacing the medium with a low serum differentiation medium $\left(2 \%\right.$ horse serum; Gibco ${ }^{\circledR}$, Life Technologies Japan, Ltd., Tokyo) for 2 days. After 48 h, the induction medium was removed and cells were maintained in DMEM with $10 \%$ FBS and, thereafter, the medium was changed every 2 days until day 5 . Telmisartan was dissolved in dimethyl sulfoxide (DMSO) and added to culture media within $0.1 \%$ of volume (telmisartan final concentration: $0-1 \mu \mathrm{M})$. Telmisartan treatment was performed on day 5 .

\section{$\mathrm{NAD}^{+} / \mathrm{NADH}$ measurements}

$\mathrm{NAD}^{+}$and $\mathrm{NADH}$ nucleotides were measured with an enzymatic NADH recycling assay, using the NAD +/NADH Quantification kit (BioVision, Mountain View, 
CA) following the manufacturer's recommended protocol [24]. C2C12 myotubes were collected in $200 \mu \mathrm{l}$ of $\mathrm{NADH} / \mathrm{NAD}^{+}$extraction buffer by subjecting the cells to 2 cycles of freeze/thaw. The supernatants were divided into 2 sets: to detect NADH, 1 set of supernatants was heated to $60^{\circ} \mathrm{C}$ for $30 \mathrm{~min}$ to decompose $\mathrm{NADH}$, while those from the other set was used to measure the total NADH plus $\mathrm{NAD}^{+}$-content, by performing the cycling assay in the absence of thermal decomposition. Then, the $\mathrm{NAD}^{+} / \mathrm{NADH}$ ratio was calculated.

\section{Semi-quantitative RT-PCR analysis}

Total RNA was isolated from $\mathrm{C} 2 \mathrm{C} 12$ myotubes using RNAiso plus (Takara Bio Inc., Shiga, Japan). Complementary DNA synthesis was performed, using a QuantiTect ${ }^{\circledR}$ Reverse Transcription kit (SA Biosciences, Boston, MA), from $1 \mu \mathrm{g}$ of the extracted total RNA. Then, we performed real-time RT-PCR with gene-specific primers and SYBR green dye using an Applied Biosystems 7500 Real-Time PCR System (Life Technologies Japan Ltd, Tokyo). The forward (fwd) and reverse (rev) primer sequences were as follows: Sirt1 (fwd: 5'-TGCAGACGTGGTAATGTCCA AAC-3'; rev: 5'-ACATCTTGGCAGTATTTGTGGTGA A-3'), GLUT4 (fwd: 5'-TTTCCAGCAGATCGGCTCTG ACGA-3'; rev: 5'-TAGCCAAACTGAAGGGAGCCAAG C-3'), ACO (fwd: 5'-GGTTGTCATCGCTTTGGTGCC TGT-3'; rev: $5^{\prime}$-TAACTCTGGATTGAAGGTGGCGGC G-3'), FATP-1 (fwd: 5'-CGGTGTGGTGGCTGCTCTTC TCAA-3'; rev: 5'-CGCTGCCATCTCCCCGCCATAAA T-3'), GAPDH (fwd: 5'-CAAGGTCATCCATGACAACTT TG-3'; rev: 5'-GGCCATCCACAGTCTTCTGG-3'). The reaction mixture containing reverse-transcribed cDNAs was preheated for $10 \mathrm{~min}$ at $95^{\circ} \mathrm{C}$ to activate the Taq polymerase. Fifty cycles of PCR, each consisting of a 10-s denaturation step at $95^{\circ} \mathrm{C}$, a 15 -s annealing step at $60^{\circ} \mathrm{C}$, and a 15 -s extension step at $72^{\circ} \mathrm{C}$, was then performed. Throughout real-time PCR analysis, product identities were confirmed by melting curve analysis. The quantification of each gene was determined relative to a standard curve generated from a serially diluted sample. The ratios of the amounts of target mRNA to the amount of the internal standard (GAPDH) mRNA was determined as an arbitrary unit.

\section{Western blotting}

Whole cell lysates for western blotting were harvested in RIPA buffer (Wako Pure Chemical Industries, Ltd., Tokyo) containing protease inhibitors (Takara Bio Inc., Shiga) and phosphatase inhibitor (Nacalai Tesque, Kyoto, Japan). Protein concentrations in the supernatants were determined using the Bio-Rad protein assay (Bio-Rad Laboratories, Inc., Tokyo). Twenty micrograms of protein were separated on a 5-20\% gradient SDS-
PAGE gel (ATTO Corporation, Tokyo) and transferred to polyvinylidene difluoride membranes (Millipore Corp., Billerica, MA). Membranes were blocked for $1 \mathrm{~h}$ at $20-25^{\circ} \mathrm{C}$ with $2 \%$ bovine serum albumin (BSA) or $5 \%$ Skim milk tris buffer saline Tween 20 (TBST) buffer. The membranes were incubated with appropriate dilutions of the primary antibodies (Toyobo Co., Ltd., Tokyo), viz., anti-Adiponectin (PA1-054 [1:2500 dilution]; Thermo Fisher Scientific Inc., Rockford, Illinois), antiPhospho-AMPKa (Thr172) (2531 [1:5000 dilution]; Cell Signaling Technology, Inc., Beverly, MA), anti-AMPK $\alpha$ (2532 [1:5000 dilution]; Cell Signaling Technology, Inc.), and anti-GAPDH (2118 [1:5000 dilution]; Cell Signaling Technology, Inc.), overnight at $4^{\circ} \mathrm{C}$. After washing, the blots were then incubated with HRP-conjugated antirabbit secondary antibody (7074 [dilution 1:5000], Cell Signaling Technology, Inc.) for $1 \mathrm{~h}$ in a room with a temperature maintained at $22 \pm 2^{\circ} \mathrm{C}$. Signals were detected using the Amersham ${ }^{\text {Tu }} E C L^{\text {Th }}$ Prime enhanced chemiluminescence system (GE Healthcare Japan, Tokyo) and LAS3000 mini imaging system (FujiFilm, Tokyo).

\section{Statistical analysis}

All data are expressed as the means \pm standard error (SE). Statistical analysis was analyzed with Student's $t$ test for parametric comparison between 2 groups, or analysis of variance (ANOVA) with post-hoc testing by Fisher's protected least significant difference (PLSD) test for multiple comparisons. Values of $\mathrm{p}<0.05$ were considered statistically significant.

\section{Results}

\section{In vivo study}

During the 5 weeks of the study period, $d b / d b$ mice fed a high-fat diet gained weight, irrespective of whether they were treated with $\mathrm{CMC}$, telmisartan, or telmisartan + GW9662 (Figures 1A and B). Systolic blood pressure remained unchanged in all groups during the study period (Figure 1C). Overnight fasting glucose levels were higher in the $d b / d b$ mice than in the wild-type mice, but were not different among the 3 treatment $d b / d b$ groups (Figure 1D). Plasma levels of adiponectin were not significantly different among groups [wild-type $5.22 \pm 0.49 \mu \mathrm{g} / \mathrm{mL}(\mathrm{n}=6)$, CMC $9.14 \pm 2.88(\mathrm{n}=5)$ telmisartan $9.09 \pm 1.07(\mathrm{n}=4)$, and telmisartan + GW9662 $6.67 \pm 0.49(\mathrm{n}=4)]$.

The ITT at the end of the 5-week study period demonstrated that the 120-min glucose levels were significantly decreased in telmisartan-treated and telmisartan + GW9662-treated mice as compared to CMC-treated mice (Figure 1E). The baseline insulin levels, measured before the ITT, had been comparable among the $3 d b / d b$ groups (Figure 1F). 

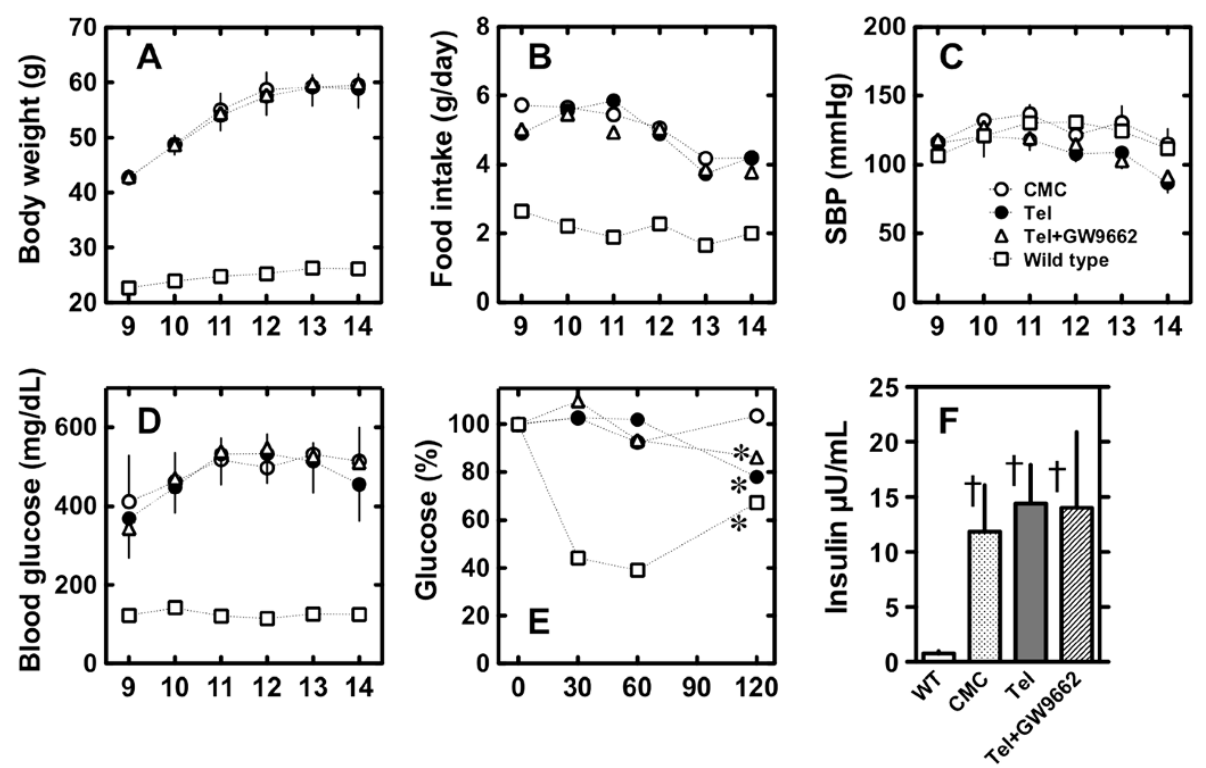

Figure 1 Effects of telmisartan on $d b / d b$ mice fed a high-fat diet. In $d b / d b$ mice fed a high-fat diet with $C M C(O, n=6)$, telmisartan $(\cdot, n=6)$, or telmisartan + GW9662 $(\Delta, n=7)$ for 5 weeks, body weight $(\mathbf{A})$, food intake (B), systolic blood pressure (C), blood glucose (D), glucose levels during the insulin tolerance test $(\mathbf{E})$, and fasting insulin levels $(\mathbf{F})$ were measured. Values for wild type mice fed normal chow $(\square, n=7)$ are also shown for reference. Glucose levels during the insulin tolerance test were measured after intraperitoneal injection with $0.75 \mathrm{U} / \mathrm{kg}$ insulin. Values shown represent means \pm S.E. ${ }^{*} \mathrm{p}<0.05$ vs. 0 min and $t p<$ wild type, by Fisher's PLSD.

\section{Effects of telmisartan on Sirt1, PGC1a, and GLUT4 mRNA expression}

In skeletal muscle, expression of Sirt1 mRNA was increased in telmisartan-treated mice compared to CMC-treated mice, but the expression was similar in telmisartan- and telmisartan + GW9662-treated mice (Figure 2). Expression of $P G C 1 \alpha$ and GLUT4 mRNA was not different between CMC-treated and telmisartan-treated mice.

In visceral adipose tissue (VAT) and subcutaneous adipose tissue (SAT), expression of Sirt1, PGC1 $\alpha$, and GLUT4 mRNA were profoundly decreased in CMCtreated $d b / d b$ mice as compared to wild type. The expression was comparable between telmisartan-treated and telmisartan + GW9662-treated mice.

\section{Effects of telmisartan on the size of adipocytes and} pancreatic islets in $d b / d b$ mice fed a high-fat diet

The mean size of adipocyte in VAT and SAT was $\approx 2$ times larger in $\mathrm{db} / \mathrm{db}$ mice as compared to wild type (mean size of VAT $88 \mu \mathrm{m}$ and SAT $94 \mu \mathrm{m}$ ). There were no significant differences in the size distribution (Figure 2) and the mean size among CMC-, telmisartan-, and telmisartan + GW9662-treated mice: $171 \mu \mathrm{m}$, $179 \mu \mathrm{m}$, and $182 \mu \mathrm{m}$ in VAT and $181 \mu \mathrm{m}, 180 \mu \mathrm{m}$, and $178 \mu \mathrm{m}$ in SAT. However, although pancreatic islet size was increased in CMC-treated compared to wild-type mice, it was significantly decreased in telmisartan- and telmisartan + GW9662-treated mice (Figure 3).

\section{Effect of telmisartan on AMPK-SIRT1 pathway}

Since telmisartan increased Sirt1 mRNA levels in the skeletal muscle of $d b / d b$ mice, we investigated whether telmisartan administration could directly affect SIRT1 signals in $\mathrm{C} 2 \mathrm{C} 12$ myoblasts. Treatment with telmisartan increased Sirt1 mRNA in C2C12 cells (Figure 4A). Treatment of $\mathrm{C} 2 \mathrm{C} 12$ cells with telmisartan resulted in activation of AMPK, by means of phosphorylation, to a level comparable to that induced by 5 -aminoimidazole4-carboxamide ribonucleoside (AICAR), an activator of AMPK (Figure 4B). Treatment of C2C12 cells with telmisartan increased $\mathrm{NAD}^{+}$and decreased $\mathrm{NADH}$, resulting in an increase in the $\mathrm{NAD}^{+} / \mathrm{NADH}$ ratio, to a level comparable to that induced by AICAR (Figure 4C).

\section{Effect of telmisartan on mRNA expression of AMPK/SIRT1} pathway-related molecules in $\mathrm{C} 2 \mathrm{C} 12$ myocytes

Treatment with telmisartan increased the mRNA levels of PGC1, FATP1, ACO and GLUT4, but not of GLUT1, in $\mathrm{C} 2 \mathrm{C} 12$ myocytes (Figure 5).

\section{Discussion}

Here, we showed that telmisartan treatment improved insulin sensitivity in obese $d b / d b$ mice fed a high-fat diet and led to reduction in the size of hypertrophic pancreatic islets in these mice. Moreover, we demonstrated that in vitro treatment with telmisartan led to increased expression of Sirt1 mRNA in skeletal muscle cells; the increase in Sirt1 mRNA in telmisartan-treated $\mathrm{C} 2 \mathrm{C} 12$ 

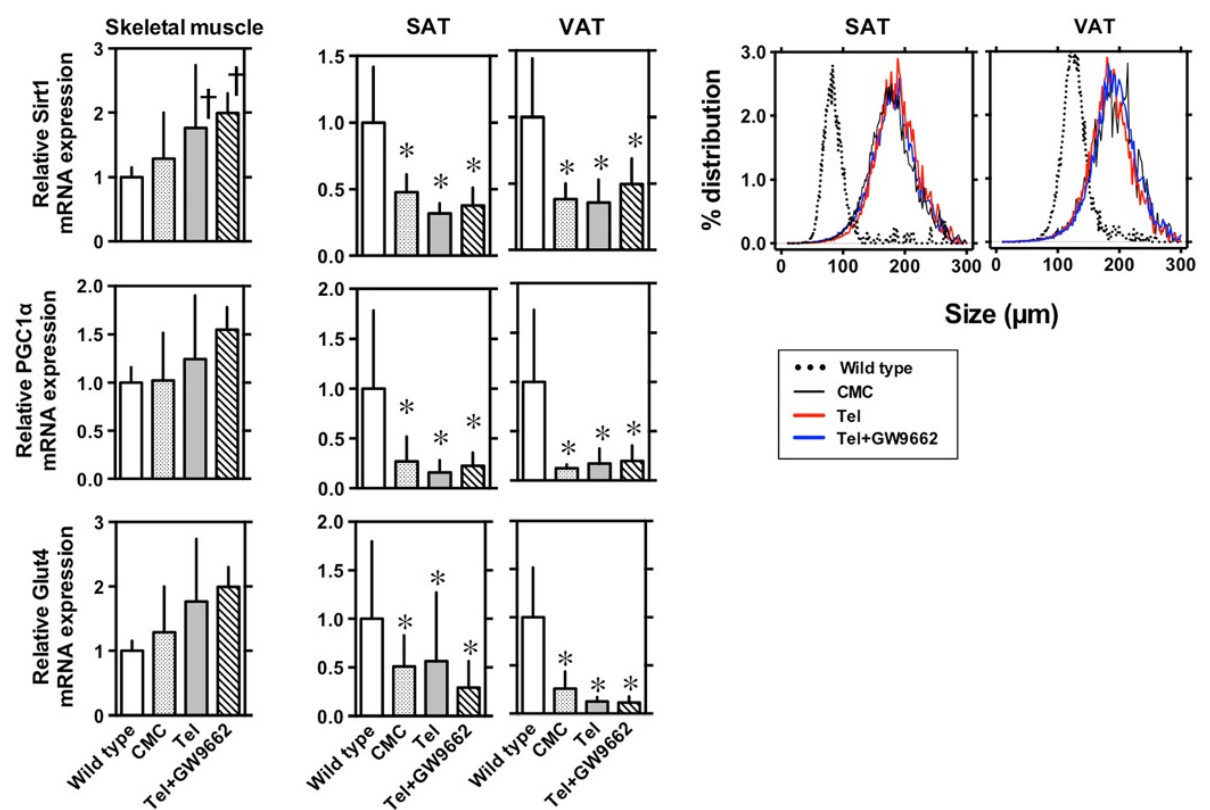

Size $(\mu \mathrm{m})$
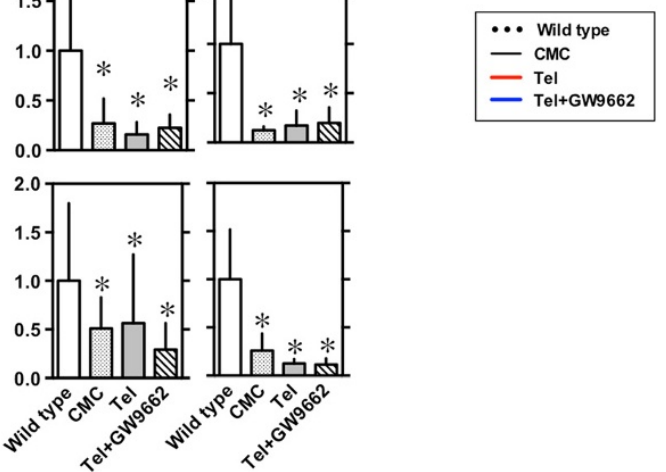

Figure 2 Effects of telmisartan on mRNA expression and adipocyte size distribution in $d b / d b$ mice fed a high-fat diet. At sacrificed after 5-week treatment, Sirt1, PGCla, and GLUT4 mRNA expression were semi-quantified by RT-PCR in skeletal muscle and in visceral adipose tissue (VAT) and subcutaneous adipose tissue (SAT). GAPDH was used as an internal control. Distribution of adipocyte size was determined by the Coulter Multisizer III (Beckman Coulter, High Wycombe, England) after fixed with 2\% osmium tetroxide. Values shown represent means \pm S.E $(n=3-4) .{ }^{*} p<0.05$ vs. wild type and $t p<0.05$ vs. CMC by Fisher's PLSD.

myoblasts occurred concomitantly with an increase in AMPK phosphorylation, an increase in $\mathrm{NAD}^{+} / \mathrm{NADH}$ ratio, and increases in the mRNA levels of PGC1, FATP1, ACO, and GLUT4.

It has previously been shown that levels of Sirt1 mRNA and SIRT1 activity are decreased in an animal obesity model $[25,26]$ and in obese humans [27]. To investigate the potential consequences of decreased SIRT1, we utilized $d b / d b$ mice, a genetic model of obesity and insulin resistance resulting from a leptin receptor mutation [28]. On a high-fat diet, this $d b / d b$ strain demonstrates severe insulin resistance and hyperglycemia [29]. In the current study, all mice fed a high-fat diet gained body weigh comparably (Figures 1A and B) and casual glucose levels did not differ among the 3 treatment groups (Figure 1D), but ITT-determined glucose levels were significantly decreased in telmisartan-treated mice. This indicates that administration of telmisartan ameliorates insulin resistance in the obesity and insulin resistance model. Because co-administration of GW9662, a selective PPAR $\gamma$ inhibitor, could not reverse this in vivo effect of telmisartan, the effects of telmisartan appear to act via a PPAR $\gamma$ independent pathway. It has been suggested that some of angiotensin II receptor blockers (ARBs) have beneficial effects on lipid metabolism [30] and insulin sensitivity possibly through PPAR- $\gamma$ activation [9-13]. We confirmed that 0.5-10 $\mu \mathrm{M}$ of telmisartan, but not up to $10 \mu \mathrm{M}$ of olmesartan nor valsartan, have a PPAR- $\gamma$ agonistic effect in HEK293 cells (data not shown). It should be clarified how different
ARBs show mechanism(s) beyond blockade of reninangiotensin system (RAS) in future studies.

Since it has been reported that AMPK activity and SIRT1 expression are decreased in the white adipose tissue (WAT) of $d b / d b$ and HFD mice [26], we evaluated mRNA levels of Sirt1, PGC1 $\alpha$, and GLUT4 in the VAT and SAT of the various $d b / d b$ mouse treatment groups. Consistent with a previous study [26], vehicle-treated $d b / d b$ mice showed a decreased expression of Sirt1, PGC1 $\alpha$, and GLUT4 mRNAs in the VAT and SAT. Moreover, treatment with telmisartan did not change these expression levels, suggesting that telmisartan improved insulin resistance via a non-adipose tissue metabolic network in this $d b / d b$ model. This hypothesis is supported by the finding that the adipocyte size distribution was not changed by telmisartan treatment. However, telmisartan administration did upregulate expression of Sirt1 mRNA and levels of phospho-Thr172-AMPK in skeletal muscle.

Metabolic sensors such as AMPK and SIRT1 are the gatekeepers of the activity of the master regulator, the mitochondrion, and are crucial to the regulatory network for metabolic homeostasis [1,31,32]. Mice models mildly overexpressing SIRT1 demonstrated ameliorated glucose tolerance when insulin resistance and/or diabetes were induced [33,34]. Similarly, treatment with different SIRT1 agonists prevents weight gain and insulin resistance when mice were challenged with high-fat diets $[8,35]$. Similarly, pharmacological activation of AMPK by 

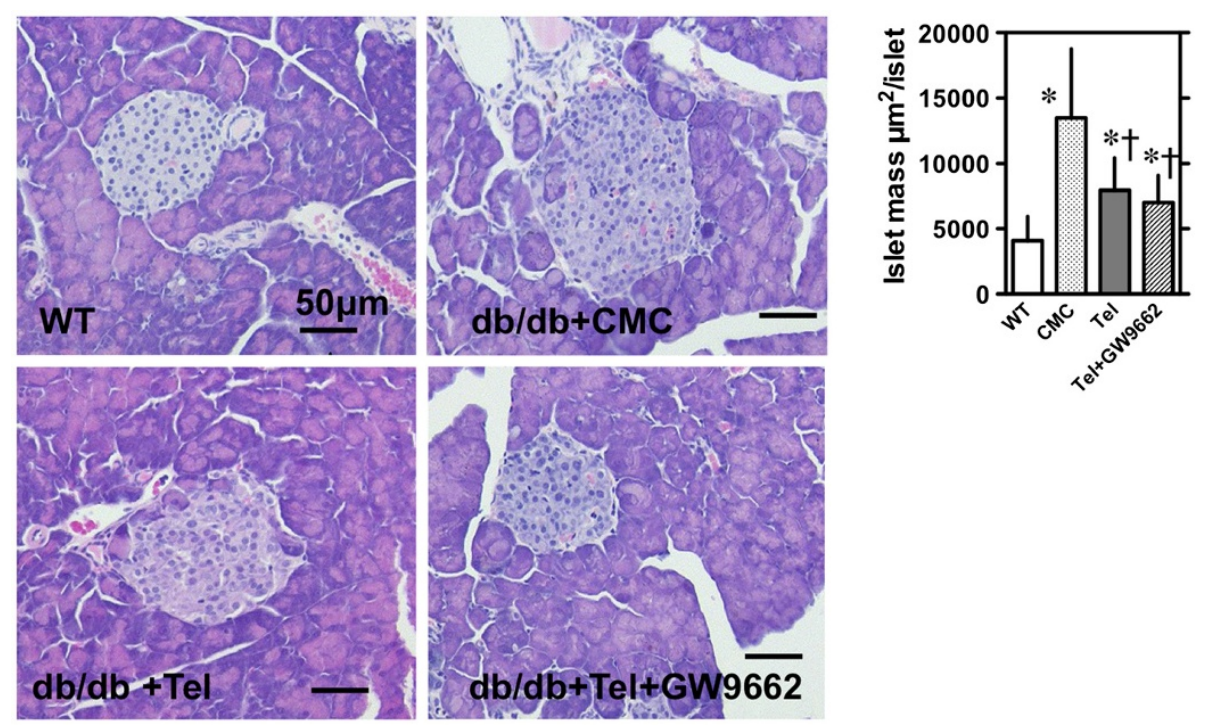

Figure 3 Effects of telmisartan on the morphology and mass of pancreatic islets in $d b / d b$ mice fed a high-fat diet. Representative sections of paraffin-embedded serial sections of pancreata (5- $\mu \mathrm{m} \mathrm{m}$ thickness) in wild type mice or $d b / d b$ mice fed a high-fat diet with CMC, telmisartan, or telmisartan + GW9662 were stained for hematoxylin and eosin (HE). The size of pancreatic islets was determined from manually traced islet area. Values shown represent means \pm S.E $(n=115-150)$. $t p<0.05$ vs. CMC by Fisher's PLSD.

metformin [6] and A-769662 [7] ameliorated insulin resistance in animals fed a high-fat diet. These results indicate a role for AMPK/SIRT1 in the control of metabolic homeostasis. It is believed that metformin treatment is the successful approach to reduce insulin resistance mainly through activation of liver AMPK [6], whereas DPP-4 inhibitors exhibit the best effects after oral glucose loading, emphasizing the incretin effect after oral stimulation [36]. However, metformin and DPP-4 inhibitors may involve different mechanisms in the treatment of mice fed high-fat diet [37]. Bourron et al. suggested a possible role of metformin on AMPK dependent lipolysis in adipocytes that may lead to lower plasma levels of fatty acids and to alleviating insulin

\section{A. Sirt1 mRNA}

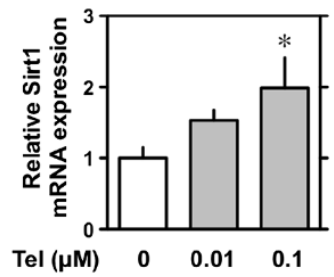

\section{B. AMPK western blot}

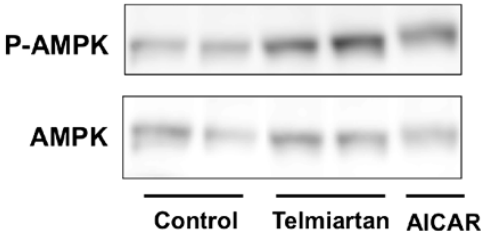

C. NAD, NADH and NAD/NADH
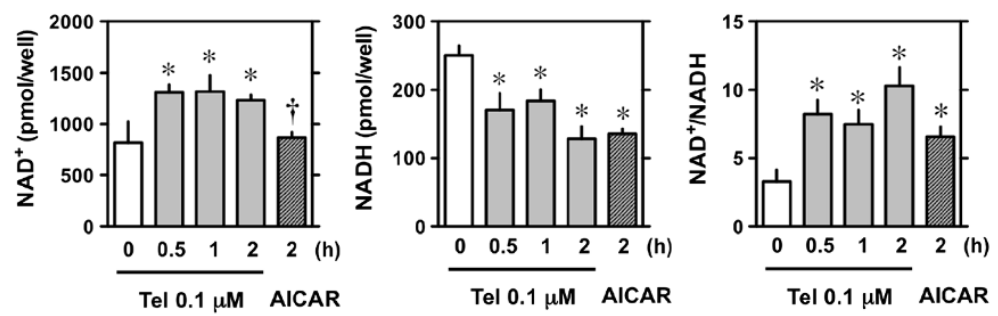

Figure 4 Effects of telmisartan on the AMPK/SIRT1 pathway in C2C12 myocytes. C2C12 myocytes were treated with vehicle or $0.01-0.1 \mu M$ telmisartan and the level of Sirt1 mRNA measured by RT-PCR (A). On day 5, C2C12 myocytes were treated with $0.1 \mu \mathrm{M}$ telmisartan or $2 \mathrm{mM}$ 5-aminoimidazole-4-carboxamide ribonucleoside (AICAR), an activator of AMP-activated protein kinase (AMPK), for 60 min, after which total cell lysates were subjected to western blot analysis using antibodies specific for Phospho-AMPK and total AMPK (B). NAD + NADH content, and NAD ${ }^{+} / N^{\prime} A D H$ ratio (C) in C2C12 myocytes treated with telmisartan or AICAR ( $0.5 \mathrm{mM})$. Values shown represent means \pm S.E $(n=3-5)$. * $p<0.05$ vs. 0 , by Fisher's PLSD. 

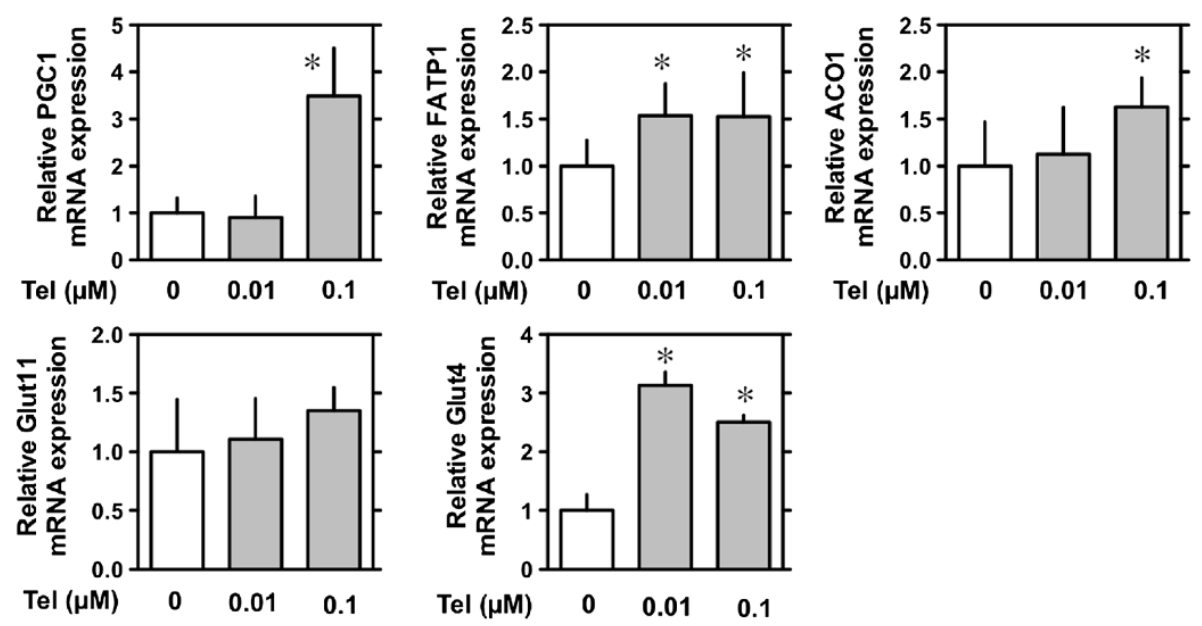

Figure 5 Effects of telmisartan on AMPK/SIRT1 pathway in C2C12 myocytes. On day 5, C2C12 myocytes were treated with telmisartan (indicated concentrations) for $12 \mathrm{~h}$ and analyzed by semi-quantitative RT-PCR. GAPDH was used as an internal control. Values shown represent the means \pm S.E $(n=3-5) .{ }^{*} p<0.05$ vs. $0 \mu M$ or baseline, by Fisher's PLSD.

resistance [38]. Sitagliptin also decreased adipocyte size efficiently and, even though the precise mechanisms remain to be elucidated, DPP-4 inhibition produced extrapancreatic effects, such as enhanced postprandial lipid mobilization and oxidation [36]. Altogether, upregulation of Sirt1 mRNA and activation of AMPK in the skeletal muscle are associated with the improved insulin sensitivity observed upon telmisartan treatment.

PGC1 $\alpha$ and GLUT4 mRNA expression also tended to be increased in skeletal muscle cells. AMPK and SIRT1 have both been described to directly affect PGC-1 $\alpha$ activity through phosphorylation and deacetylation, respectively [32]. Thus, we next evaluated the direct effect of telmisartan on PGC- $1 \alpha$ activity in muscle using

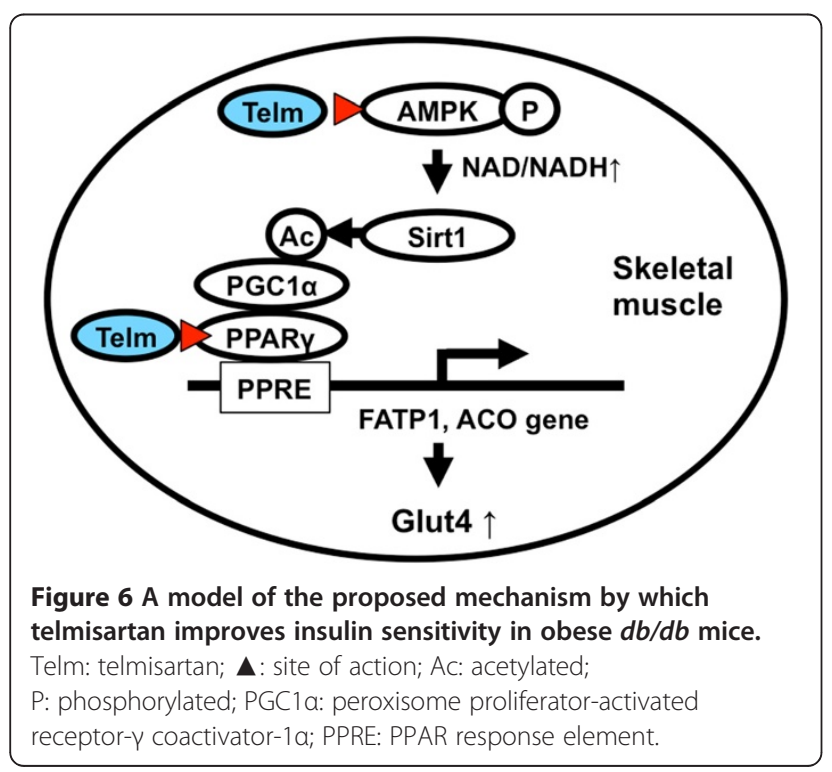

C2C12 mouse myoblast cells. We found that in vitro treatment with telmisartan increased Sirt1 mRNA concomitantly with the increase in AMPK phosphorylation, an increase in the $\mathrm{NAD}^{+} / \mathrm{NADH}$ ratio, and the mRNA levels of PGC1, FATP1, ACO, and GLUT4 in C2C12 cells. To our knowledge, the current study, for the first time, showed that treatment with telmisartan enhanced Sirt1 mRNA and protein in the skeletal muscle.

SIRT1, an $\mathrm{NAD}^{+}$-dependent regulator of energy metabolism, has been reported to be activated through AMPK-mediated induction of nicotinamide phosphoribosyltransferase (NAMPT), the rate-limiting enzyme for $\mathrm{NAD}^{+}$biosynthesis $[1,8]$. We thus examined the direct effects of telmisartan on AMPK. Telmisartan resulted in a significant increase in AMPK $\alpha$ phosphorylation at Thr172, to a level comparable to that induced by AICAR, an activator of AMPK.

Taken altogether, we propose a possible mechanism by which telmisartan improves insulin sensitivity in skeletal muscle in Figure 6D. Impaired adipocytokine signaling, caused by suppression of expression of leptin and adipoR2 receptors, could lead to decreased activation of AMPK. This, in turn, may result in decreased NAMPT transcription, and consequently decreased SIRT1 activity and expression. Our results are also consistent with the notion that telmisartan, besides blocking AT1, acts to promote adipocyte differentiation and improve insulin sensitivity.

As previously reported, pancreatic islets in $d b / d b$ mice on a high-fat diet were profoundly hypertrophic, presumably as compensation for severe insulin resistance. Saitoh et al. reported that telmisartan decreased the accumulation of palmitate-induced reactive oxygen species in MIN6 cells by $25 \%$, and by $55 \%$ in mouse islet cells, suggesting that RAS blockade may be a possible 
mechanism for protecting $\beta$-cell survival and preserving insulin secretion capacity [39]. Yuan et al. reported that telmisartan and perindopril, a RAS inhibitor, comparably improved islet morphology, and significantly reduced $I L-1 \beta, H I F-1 \alpha$, and CHOP mRNA in Wistar rats fed a high-fat diet for 16 weeks [40]. They suggested that blockade of RAS may protect the islet function of rats on a long-term high-fat diet, via down-regulation of islet inflammation, oxidative stress, endoplasmic reticulum stress, and apoptosis [41]. Our study showed that such hypertrophic pancreatic islets were improved in telmisartan-treated, and in telmisartan + GW9662-treated mice. The distribution in pancreatic $\beta$ cell mass may also be affected by telmisartan, but we could not determine variation in the $\beta$ cell mass distribution by our method. Although we could not determine the mechanism by which telmisartan affects islet morphology and function, future studies should determine the precise mechanism of the antimetabolic effects of this drug.

Our study had some limitations. First, the $\mathrm{C} 2 \mathrm{C} 12$ model has limitations in comparison to freshly prepared frozen skeletal muscle cells [23]: $\mathrm{C} 2 \mathrm{C} 12$ cells were originally obtained by Yaffe and Saxel through serial passage of myoblasts cultured from the thigh muscle of $\mathrm{C} 3 \mathrm{H}$ mice after a crush injury. $\mathrm{C} 2 \mathrm{C} 12$ cells are a useful tool for studying the differentiation of myoblasts and osteoblasts, expressing various proteins, and exploring mechanistic pathways. Nevertheless, the $\mathrm{C} 2 \mathrm{C} 12$ cell line originated from a single clone, and thus has clone-specific traits, and fails to recapitulate primary cells. Second, the use of a single concentration of GW9662 may not be specific for verifying inhibition of PPARY activity. We used a single dose of $1 \mathrm{mg} / \mathrm{kg}$ of GW9662 for the in vivo studies, and a single dose of $5 \mu \mathrm{M}$ of GW9662 for the in vitro studies. We determined the effect of telmisartan on the activity of a PPAR 2 promoter luciferase gene reporter construct, indicating that $0.5-10.1 \mathrm{mM}$ of telmisartan increased the activity of the PPAR 2 gene promoter by 1.8 - to 2 -fold, to a $\simeq 30 \%$ level comparable to that achieved by pioglitazone, a known PPAR $\gamma$ agonist (in submission). The effects of telmisartan through PPAR $\gamma$-dependent and -independent pathways need to be confirmed in further studies. Third, nonspecific effects of the drugs used in this study cannot be eliminated. GW9662 has been shown to inhibit COX-2 activity in addition to having an inhibitory effect on PPAR $\gamma$, the active derivative of AICAR modulates other AMP-sensitive enzymes, and the selectivity of compound $C$ for inhibition of AMPK remains uncertain. The findings presented in this study should be confirmed by using dominant-negative vectors or siRNA knockdown of AMPK and SIRT1 in the future. Fourth, our study could not determine whether telmisartan regulates AMPK/SIRT1 pathways in a tissueor model-specific manner. Caton et al. reported that AMPK activity, NAMPT expression and SIRT1 expression were decreased in the WAT of $d b / d b$ and HFD mice [26]. In addition, they showed that metformin increased the AMPK activity in the WAT of $d b / d b$ mice and in adipocytes treated with metformin, with increased NAMPT and SIRT1 levels. In our study, telmisartan showed significant changes in protein and mRNA levels in skeletal muscle, but not in WAT. Although we cannot explain this discrepancy between the 2 studies, Chen et al. reported that SIRT1 expression is differentially regulated, in a tissue-specific manner upon calorie restriction, which may reflect tissue-specific roles of SIRT1 [41]. Tissue-specific effects of telmisartan need to be elucidated in future studies. Finally, the estimation of cell size has a limitation: in the fat pad, central adipocytes are usually bigger than peripheral adipocytes, and islets are roughly spheres and sections could cut spheres at its poles and distort the results.

In conclusion, telmisartan treatment improved glucose intolerance in $d b / d b$ mice fed a high-fat diet, which was not inhibited by GW9662 co-administration. Telmisar$\tan$ increased Sirt1 mRNA concomitantly with activation of AMPK via phosphorylation, an increase in NAD ${ }^{+} / \mathrm{NADH}$ ratio, and increase in mRNA levels of $P G C 1 \alpha$, FATP1, ACO, and GLUT4 in C2C12 cell lines, suggesting that these improvements were at least partially due to the direct actions of telmisartan on AMPK/SIRT1 pathways. Precise understanding of this molecular mechanism will require further investigations.

\section{Abbreviations}

ANOVA: Analysis of variance; AT1: Angiotensin II type 1 receptor; DMEM: Dulbecco's modified Eagle's medium; DMSO: Dimethyl sulfoxide; G6PDH: Glucose-6-phosphate dehydrogenase; ITT: Insulin tolerance test; NAD: Nicotinamide adenine dinucleotide; PPAR: Peroxisome proliferatoractivated receptor; RT-PCR: Real-time-polymerase chain reaction;

Sirt1: Nicotinamide adenine dinucleotide (NAD)-dependent deacetylase.

\section{Competing interests}

The authors declare that they have no competing interests.

\section{Authors' contributions}

AS, MS, MS: design and conducting the study, data collection, analysis, and manuscript writing; $\mathrm{HA}, \mathrm{MH}, \mathrm{YH}, \mathrm{HK}, \mathrm{YN}, \mathrm{HM}$ : contribution to design and discussion; HA and HA: contribution to cell distribution study; NM and IS: contribution to C2C12 study. We sincerely thank Mss. ST, JK and HG for her assistance. All authors read and approved the final manuscript.

\section{Acknowledgements}

This study was supported by grants from the Ministry of Education, Culture, Sports, Science, and Technology, and the Ministry of Health, Labor, and Welfare (MHLW) and Joint Research Association for Japanese Diabetes. We thank Nippon Boehringer Ingelheim Co., Ltd., Japan, for kindly providing telmisartan.

\section{Author details}

'Department of Cardio-Diabetes Medicine, University of Tokushima Graduate School of Health Biosciences, 3-18-15 Kuramoto, Tokushima 770-8503, Japan. ${ }^{2}$ Department of Cardiovascular Medicine, University of Tokushima Graduate School of Health Biosciences, Tokushima, Japan. ${ }^{3}$ Department of Nutrition and Metabolism, University of Tokushima Graduate School of Health Biosciences, Tokushima, Japan. ${ }^{4}$ Department of Cardiovascular Surgery, University of Tokushima Graduate School of Health Biosciences, Tokushima, Japan. ${ }^{5}$ Department of Metabolic Medicine, Osaka University Graduate School of Medicine, Osaka, Japan. ${ }^{6}$ Division of Endocrinology, Diabetes and Metabolism, Hematology, Rheumatology, Second Department of Internal 
Medicine, University of the Ryukyus, Graduate School of Medicine, Okinawa, Japan.

Received: 29 August 2012 Accepted: 18 October 2012 Published: 8 November 2012

\section{References}

1. Houtkooper RH, Pirinen E, Auwerx J: Sirtuins as regulators of metabolism and healthspan. Nat Rev Mol Cell Biol 2012, 13:225-238.

2. Fulco $M$, Schiltz RL, lezzi $S$, et al: Sir2 regulates skeletal muscle differentiation as a potential sensor of the redox state. Mol Cell 2003, 12:51-62.

3. Vinciguerra M, Fulco M, Ladurner A, Sartorelli V, Rosenthal N: SirT1 in muscle physiology and disease: lessons from mouse models. Dis Model Mech 2010, 3:298-303.

4. Tonkin J, Villarroya F, Puri PL, Vinciguerra M: SIRT1 signaling as potential modulator of skeletal muscle diseases. Curr Opin Pharmacol 2012, Epub ahead of print.

5. Zhang BB, Zhou G, Li C: AMPK: an emerging drug target for diabetes and the metabolic syndrome. Cell Metab 2009, 9:407-416.

6. Ouyang J, Parakhia RA, Ochs RS: Metformin activates AMP kinase through inhibition of AMP deaminase. J Biol Chem 2011, 286:1-11.

7. Cool B, Zinker B, Chiou W, et al: Identification and characterization of a small molecule AMPK activator that treats key components of type 2 diabetes and the metabolic syndrome. Cell Metab 2006, 3:403-416.

8. Lagouge M, Argmann C, Gerhart-Hines Z, et al: Resveratrol improves mitochondrial function and protects against metabolic disease by activating SIRT1 and PGC-1alpha. Cell 2006, 127:1109-1122.

9. Benson SC, Pershadsingh $\mathrm{HA}, \mathrm{Ho} \mathrm{Cl}$, et al: Identification of telmisartan as a unique angiotensin II receptor antagonist with selective PPARgammamodulating activity. Hypertension 2004, 43:993-1002.

10. Araki K, Masaki T, Katsuragi I, Tanaka K, Kakuma T, Yoshimatsu H: Telmisartan prevents obesity and increases the expression of uncoupling protein 1 in diet-induced obese mice. Hypertension 2006, 48:51-57.

11. Sugimoto K, Qi NR, Kazdova L, Pravenec M, Ogihara T, Kurtz TW: Telmisartan but not valsartan increases caloric expenditure and protects against weight gain and hepatic steatosis. Hypertension 2006, 47:1003-1009.

12. Pershadsingh HA, Kurtz TW: Insulin-sensitizing effects of telmisartan: implications for treating insulin-resistant hypertension and cardiovascular disease. Diabetes Care 2004, 27:1015.

13. Shimabukuro M, Tanaka $H$, Shimabukuro T: Effects of telmisartan on fat distribution in subjects with the metabolic syndrome. J Hypertens 2007, 25:841-848.

14. Evans RM, Barish GD, Wang YX: PPARs and the complex journey to obesity. Nat Med 2004, 10:355-361.

15. Kurtz TW: Beyond the classic angiotensin-receptor-blocker profile. Nat Clin Pract Cardiovasc Med 2008, 5:S19-26.

16. Chaudhary N, Pfluger PT: Metabolic benefits from Sirt1 and Sirt1 activators. Curr Opin Clin Nutr Metab Care 2009, 12:431-437.

17. Sugden MC, Caton PW, Holness MJ: PPAR control: it's SIRTainly as easy as PGC. J Endocrinol 2010, 204:93-104

18. Reeves PG, Nielsen FH, Fahey GC Jr: AIN-93 purified diets for laboratory rodents: final report of the American Institute of Nutrition ad hoc writing committee on the reformulation of the AIN-76A rodent diet. J Nutr 1993, 123:1939-1951.

19. Stangier J, Su CA, Roth W: Pharmacokinetics of orally and intravenously administered telmisartan in healthy young and elderly volunteers and in hypertensive patients. J Int Med Res 2000, 28:149-67.

20. Goyal S, Arora S, Bhatt TK, Das P, Sharma A, Kumari S, Arya DS: Modulation of PPAR-gamma by telmisartan protects the heart against myocardial infarction in experimental diabetes. Chem Biol Interact 2010, 185:271-80.

21. Sakai T, Sakaue $H$, Nakamura T, Okada M, Matsuki $Y$, Watanabe E, Hiramatsu R, Nakayama K, Nakayama Kl, Kasuga M: Skp2 controls adipocyte proliferation during the development of obesity. J Biol Chem 2007, 282:2038-2046.

22. Shimabukuro $\mathrm{M}$, Ohneda $\mathrm{M}$, Lee $\mathrm{Y}$, Unger $\mathrm{RH}$ : Role of nitric oxide in obesity-induced beta cell disease. J Clin Invest 1997, 100:290-5.

23. Yaffe D, Saxel O: Serial passaging and differentiation of myogenic cells isolated from dystrophic mouse muscle. Nature 1977, 270:725-727.
24. Iwabu M, Yamauchi T, Okada-Iwabu M, et al: Adiponectin and AdipoR1 regulate PGC-1 $a$ and mitochondria by $\mathrm{Ca}^{2+}$ and AMPK/SIRT1. Nature 2010, 464:1313-1319.

25. Qiao L, Lee B, Kinney B, Yoo HS, Shao J: Energy intake and adiponectin gene expression. Am J Physiol Endocrinol Metab 2011, 300:E809-816.

26. Caton PW, Kieswich J, Yagoob MM, Holness MJ, Sugden MC: Metformin opposes impaired AMPK and SIRT1 function and deleterious changes in core clock protein expression in white adipose tissue of geneticallyobese $d b / d b$ mice. Diabetes Obes Metab 2011, 13:1097-1104.

27. Clark SJ, Falchi M, Olsson B, et al: Association of sirtuin 1 (SIRT1) gene SNPs and transcript expression levels with severe obesity. Obesity 2012, 20:178-185.

28. Lee $\mathrm{GH}$, Proenca R, Montez JM, et al: Abnormal splicing of the leptin receptor in diabetic mice. Nature 1996, 379:632-635

29. Rong JX, Qiu Y, Hansen MK, et al: Adipose mitochondrial biogenesis is suppressed in $d b / d b$ and high-fat diet-fed mice and improved by rosiglitazone. Diabetes 2007, 56:1751-1760.

30. Nishida Y, Takahashi Y, Nakayama T, Soma M, Asai S: Comparative effect of olmesartan and candesartan on lipid metabolism and renal function in patients with hypertension: a retrospective observational study. Cardiovasc Diabetol 2011, 10:74

31. Liang F, Kume S, Koya D: SIRT1 and insulin resistance. Nature Rev Endocrinol 2009, 5:367-373

32. Cantó C, Auwerx J: PGC-1a, SIRT1 and AMPK, an energy sensing network that controls energy expenditure. Curr Opin Lipidol 2009, 20:98-105.

33. Banks AS, Kon N, Knight C, et al: SirT1 gain of function increases energy efficiency and prevents diabetes in mice. Cell Metab 2008, 8:333-341.

34. Bordone $\mathrm{L}$, Cohen D, Robinson A, et al: SIRT1 transgenic mice show phenotypes resembling calorie restriction. Aging Cell 2007, 6:759-767.

35. Milne JC, Lambert PD, Schenk S, et al: Small molecule activators of SIRT1 as therapeutics for the treatment of type 2 diabetes. Nature 2007, 450:712-716.

36. Baggio LL, Drucker DJ: Biology of incretins: GLP-1 and GIP. Gastroenterology 2007, 132:2131-2157.

37. Westerink J, Visseren FU: Pharmacological and non-pharmacological interventions to influence adipose tissue function. Cardiovasc Diabetol 2011, 10:13.

38. Bourron O, Daval M, Hainault I, Hajduch E, Servant JM, Gautier JF, et al: Biguanides and thiazolidinediones inhibit stimulated lipolysis in human adipocytes through activation of AMP-activated protein kinase. Diabetologia 2010, 53:768-778.

39. Saitoh $Y$, Hongwei W, Ueno H, Mizuta M, Nakazato M: Telmisartan attenuates fatty-acid-induced oxidative stress and $\mathrm{NAD}(\mathrm{P}) \mathrm{H}$ oxidase activity in pancreatic beta-cells. Diabetes Metab 2009, 35:392-397.

40. Yuan L, Li X, Li J, Li HL, Cheng SS: Effects of renin-angiotensin system blockade on the islet morphology and function in rats with long-term high-fat diet. Acta Diabetol 2010, doi:10.1007/s00592-010-0210-8.

41. Chen D, Bruno J, Easlon E, et al: Tissue-specific regulation of SIRT1 by calorie restriction. Genes Dev 2008, 22:1753-1757.

doi:10.1186/1475-2840-11-139

Cite this article as: Shiota et al: Telmisartan ameliorates insulin sensitivity by activating the AMPK/SIRT1 pathway in skeletal muscle of obese $d b / d b$ mice. Cardiovascular Diabetology 2012 11:139.

\section{Submit your next manuscript to BioMed Central and take full advantage of:}

- Convenient online submission

- Thorough peer review

- No space constraints or color figure charges

- Immediate publication on acceptance

- Inclusion in PubMed, CAS, Scopus and Google Scholar

- Research which is freely available for redistribution 